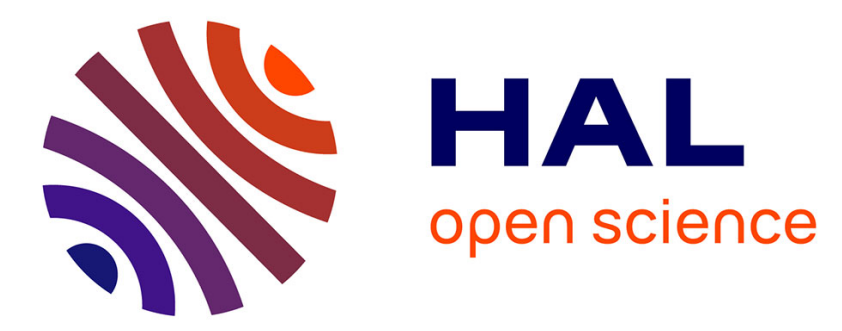

\title{
Floquet thermalisation in a Rydberg-blockaded atomic chain subject to a frequency-modulated drive
}

\author{
Jean Brion, Etienne Brion
}

\section{To cite this version:}

Jean Brion, Etienne Brion. Floquet thermalisation in a Rydberg-blockaded atomic chain subject to a frequency-modulated drive. Journal of Physics B: Atomic, Molecular and Optical Physics, inPress, 53 (10), pp.105001. 10.1088/1361-6455/ab7880 . hal-02486540

\section{HAL Id: hal-02486540 \\ https://hal.science/hal-02486540}

Submitted on 26 Feb 2020

HAL is a multi-disciplinary open access archive for the deposit and dissemination of scientific research documents, whether they are published or not. The documents may come from teaching and research institutions in France or abroad, or from public or private research centers.
L'archive ouverte pluridisciplinaire HAL, est destinée au dépôt et à la diffusion de documents scientifiques de niveau recherche, publiés ou non, émanant des établissements d'enseignement et de recherche français ou étrangers, des laboratoires publics ou privés. 


\title{
Floquet thermalisation in a Rydberg-blockaded atomic chain subject to a frequency-modulated drive
}

\author{
J. Brion* and E. Brion ${ }^{1}$ \\ ${ }^{1}$ Laboratoire Collisions Agrégats Réactivité, IRSAMC \& UMR5589 du CNRS, Université de \\ Toulouse III Paul Sabatier, F-31062 Toulouse Cedex 09, France. \\ E-mail: etienne.brion@irsamc.ups-tlse.fr
}

\begin{abstract}
We report on numerical simulations demonstrating the emergence of stroboscopic thermalisation in a chain of atoms submitted to a laser field whose frequency is periodically modulated close to resonance with a transition towards a Rydberg state. We relate the conditions of equilibration of the Rydberg population to the spectrum of the Floquet Hamiltonian and suggest a possible experimental implementation.
\end{abstract}

Keywords: Rydberg atoms, Rydberg blockade, Floquet thermalisation in closed quantum systems Submitted to: J. Phys. B: At. Mol. Phys. 


\section{Introduction}

For the last two decades, Rydberg atoms [1] have been the subject of an intense research activity, mainly due to their potential use in quantum technologies $[2,3]$. This interest is motivated by the so-called Rydberg blockade which results from the strong dipole-dipole interactions that two atoms exhibit when prepared in a highly excited level $[4,5]$. Since its discovery, Rydberg blockade was put forward as a powerful way to create entanglement among information-carrying atoms $[6,7]$ and therefore implement quantum computation [5, 8, 9], communication [10-12] and simulation protocols $[13,14]$. The highly non-linear optical response of Rydberg-excited atomic media and its applications were also addressed experimentally and theoretically, either in free-space or in cavity qed setups [15-26].

Rydberg atoms furthermore appeared as versatile systems to investigate fundamental paradigmatic problems, such as quantum dynamics after a quench. Atomic ensembles suddenly submitted to a resonant laser excitation towards a Rydberg level were indeed numerically shown to thermalise $[27,28]$ : the expectation values of certain observables - specifically single-particle ones such as the populations - exhibit rapidly damped oscillations before entering a thermal regime, in which they slightly fluctuate around an average thermal-like value [29-36]. This effect appears in simulations though the model does not include any coupling to the environment: Rydberg levels being long-lived [1], their spontaneous emission may indeed be safely neglected on the typical timescales of experiments and the atomic system may therefore satisfactorily be considered closed. Here, equilibration is fundamentally due to the dephasing process resulting from the combined action of single-particle laser excitation and long-range two-particle dipole-dipole interactions. To be more explicit, the (typically factorized) state of the ensemble just before the quench decomposes on a large number of eigenstates of the quenched Hamiltonian: if the associated eigenenergies are distributed in a sufficiently wide range, the different eigencomponents in the system state vector dephase, hence the observed effective thermalisation. According to the Eigenstate thermalisation Hypothesis - ETH [35], the reached thermalised state can be described by a canonical thermal ensemble.

Periodically driven (so-called Floquet) systems, may also exhibit stroboscopic thermalisation. In other words, the discrete-time expectation values of observables measured every period may converge towards a thermalised value, thus implying a synchronization of the system with the drive [37]. Floquet thermalisation was investigated in various systems and models, including interacting fermions [38], driven Hubbard model [39], and the $O(N)$ model [40]. Other behaviours may arise in Floquet systems, including the emergence of Floquet time crystals [41], which exhibit macroscopic oscillations at integer multiples of the driving period. Floquet stroboscopic evolution can also be specifically engineered in order to emulate exotic phases of matter [42, 43] or light [44]. Recently, a protocol for a Rydberg-based Floquet time crystal has been theoretically suggested [45] and analyzed and the implementation of interacting Floquet Rydberg cavity polaritons has been demonstrated [46].

The present work focuses on Floquet thermalisation in a chain of atoms excited towards a Rydberg level by a frequency-modulated laser beam. Here, we simulate the stroboscopic dynamics of the population in the Rydberg state, analyze the conditions for its equilibration and numerically investigate the influence of the different parameters of the system. We also present a potential implementation of our scheme in a system of atoms trapped in optical tweezers.

The article is structured as follows. In Sec. II, we briefly recall basic notions of Floquet systems and thermalisation, and introduce useful notations. In Sec. III, we present our model, provide 
numerical simulations and interpret our results. In Sec. IV we conclude and give perspectives of our work.

\section{Stroboscopic thermalisation of a closed system submitted to a periodic drive}

In this section, we introduce the general notions and notations which will be used in the remainder of the article. To this end, we consider a generic many-body closed system of natural Hamiltonian $H_{0}$, initially prepared in the state $\left|\Psi_{0}\right\rangle$. At time $t=0$, a $T$-periodic time-dependent drive $V(t)$ is switched on: the full Hamiltonian of the system $H=H_{0}+V$ is therefore itself $T$-periodic, i.e.

$$
H(t+T)=H(t)
$$

After one period, the system is in the state $\left|\Psi_{1}\right\rangle \equiv U_{F}\left|\Psi_{0}\right\rangle$ where $U_{F} \equiv \mathscr{T}\left\{\exp \left[-\frac{\mathrm{i}}{\hbar} \int_{0}^{T} \mathrm{~d} t H(t)\right]\right\}$ is the evolution operator over one period and $\mathscr{T}$ is the chronological product. As a unitary operator, $U_{F}$ can be put under the diagonal form $U_{F}=\sum_{\alpha} \exp \left(-\mathrm{i} \omega_{\alpha} T\right)|\alpha\rangle\langle\alpha|$ where $\exp \left(-\mathrm{i} \omega_{\alpha} T\right)$ and $|\alpha\rangle$ denote the eigenvalues and eigenstates of $U_{F}$, respectively, with $\omega_{\alpha} T$ uniquely determined in the interval $[-\pi, \pi]$. The Floquet Hamiltonian $H_{F}$ can then be uniquely and unambiguously defined by $H_{F} \equiv \sum_{\alpha} \hbar \omega_{\alpha}|\alpha\rangle\langle\alpha|$. The evolutions induced by $H$ and $H_{F}$ at an arbitrary time $t$ are a priori different but they do coincide at times equal to multiples of the period $T$, i.e. at $t_{n}=n T$ where $n \in \mathbb{N}$.

For an arbitrary observable $O$ of the system, expectation values at times $n T$ (for $n \in \mathbb{N}$ ), denoted by $\mathscr{O}_{n} \equiv\langle O\rangle(n T)$, therefore simply write

$$
\mathscr{O}_{n}=\left\langle\Psi_{0}\left|\left(U_{F}^{\dagger}\right)^{n} O\left(U_{F}\right)^{n}\right| \Psi_{0}\right\rangle=\sum_{\alpha \beta} e^{\mathrm{i} n T\left(\omega_{\alpha}-\omega_{\beta}\right)} c_{\alpha}^{*} c_{\beta} \mathscr{O}_{\alpha \beta}
$$

where $\mathscr{O}_{\alpha \beta} \equiv\langle\alpha|O| \beta\rangle$ and $c_{\alpha} \equiv\left\langle\alpha \mid \Psi_{0}\right\rangle$ is the projection onto the eigenvector $|\alpha\rangle$ of the initial state of the system - just before switching the drive on. Though the continuous-time function $\langle O\rangle(t)$ should not be a priori expected to thermalise - the system is subject to a time-dependent drive $\mathscr{O}_{n}$ 's may equilibrate with increasing $n$ 's, a phenomenon referred to as Floquet thermalisation.

As noted above, $\mathscr{O}_{n}$ 's are expectation values of the observable $O$ measured at discrete times $n T$ in the system of interest evolving either with the original time-dependent Hamiltonian $H(t)$ or with the constant Floquet Hamiltonian $H_{F}$. To be more explicit, denoting by $\langle O\rangle_{F}$ the expectation value of $O$ for the system evolving with $H_{F}$, one has $\mathscr{O}_{n}=\langle O\rangle(n T)=\langle O\rangle_{F}(n T)-$ though for an arbitrary time $t,\langle O\rangle(t) \neq\langle O\rangle_{F}(t)$. A sufficient condition for the stroboscopic thermalisation of $\mathscr{O}_{n}$ 's is therefore the continuous-time thermalisation of $\langle O\rangle_{F}(t)$ which can be analyzed in the formalism developed in [36].

After [36], we introduce the frequency gaps

$$
G_{\alpha \beta} \equiv\left(\omega_{\alpha}-\omega_{\beta}\right)
$$

their respective amplitudes

$$
v_{O, \alpha \beta} \equiv \frac{c_{\alpha}^{*} c_{\beta} \mathscr{O}_{\alpha \beta}}{\Delta_{O}}
$$

where $\Delta_{O}$ is the difference between the highest and lowest eigenvalues of $O$, and their respective relevances

$$
q_{O, \alpha \beta} \equiv \frac{\left|v_{O, \alpha \beta}\right|^{2}}{\sum_{a b}\left|v_{O, a b}\right|^{2}}
$$


When the gaps $G_{\alpha \beta}$ which contribute to the sum in equation (1), i.e. whose relevances $q_{O, \alpha \beta}$ do not vanish, distribute on a sufficiently large range $\Delta G$ and in a sufficiently homogeneous manner without degeneracy, the different spectral components in equation (1) dephase and interfere destructively. For $t \geq \bar{t} \approx 2 \pi / \Delta G$, i.e. for a number of periods $n \geq \bar{n}=[2 \pi / \Delta G \times T]$ - where [.] denotes the integer part - the expectation value $\mathscr{O}_{n}$ equilibrates around the value

$$
\overline{\mathscr{O}} \equiv \sum_{\alpha}\left|c_{\alpha}\right|^{2} \mathscr{O}_{\alpha \alpha}
$$

which can be seen as the mean value $\operatorname{Tr}[\bar{\rho} O]$ calculated in the mixed state $\bar{\rho} \equiv \sum_{\alpha}\left|c_{\alpha}\right|^{2}|\alpha\rangle\langle\alpha|$ also called the thermalised state. Fluctuations of $\mathscr{O}_{n}$ around $\overline{\mathscr{O}}$ can be characterized by their variance $\sigma_{O, n}^{2} \equiv \frac{1}{n} \sum_{j=0}^{n}\left(\mathscr{O}_{j}-\overline{\mathscr{O}}\right)^{2}$ and its long-time limit

$$
\bar{\sigma}_{O}^{2} \equiv \sigma_{O}^{2}(n \rightarrow+\infty)=\sum_{\alpha \neq \beta}\left(c_{\alpha}^{*} c_{\beta} \mathscr{O}_{\alpha \beta}\right)^{2}
$$

In the next section, we apply the formalism introduced above to a linear chain of atoms submitted to a laser field whose frequency is periodically modulated close to resonance with a transition towards a Rydberg state.

\section{Floquet thermalisation in a Rydberg-blockaded atomic chain}

\subsection{Model and assumptions}

In this section, we consider a system comprising $N$ atoms with a two-level structure involving a ground and a Rydberg states, respectively denoted by $|g\rangle$ and $|r\rangle$ (see figure 1 a). The atoms are regularly arranged along a line with the step $a$. They are submitted to a laser beam which near-resonantly drives the transition $|g\rangle \leftrightarrow|r\rangle$ with the Rabi frequency $\Omega$ and the time-dependent $T$-periodic detuning $\Delta(t)$. Atoms excited in the Rydberg state moreover exhibit strong dipoledipole interactions leading to the so-called Rydberg blockade [2-5]. In the usual rotating wave approximation, the full time-dependent Hamiltonian takes the form $H(t)=H_{0}+V(t)$ with

$$
\begin{aligned}
H_{0} & \equiv \frac{C_{6}}{a^{6}} \sum_{m \neq n} \frac{\sigma_{r r}^{(m)} \sigma_{r r}^{(n)}}{|m-n|^{6}} \\
V(t) & \equiv \hbar \sum_{n}\left[\Delta(t) \sigma_{r r}^{(n)}+\frac{\Omega}{2}\left(\sigma_{r g}^{(n)}+\sigma_{g r}^{(n)}\right)\right]
\end{aligned}
$$

where $\sigma_{k l}^{(m)} \equiv \mathbb{I} \otimes \cdots \otimes|k\rangle_{m}\langle l| \otimes \cdots \otimes \mathbb{I}$ is the operator $\sigma_{k l} \equiv|k\rangle\langle l|$ acting on the $m^{\text {th }}$ atom in the chain, and $C_{6}$ denotes the coefficient of the van der Waals interaction between two atoms in the state $|r\rangle$.

Emission from the Rydberg state can usually be neglected provided experimental runs are short enough. For instance, for $n>50$, the $s, p, d$, and $f$ states of rubidium have typical lifetimes (including radiative and blackbody effects) greater than about $50 \mu$ s at room temperature while experiment sequences can be achieved on the MHz-scale [2]. We therefore shall not consider spontaneous emission in our model. 


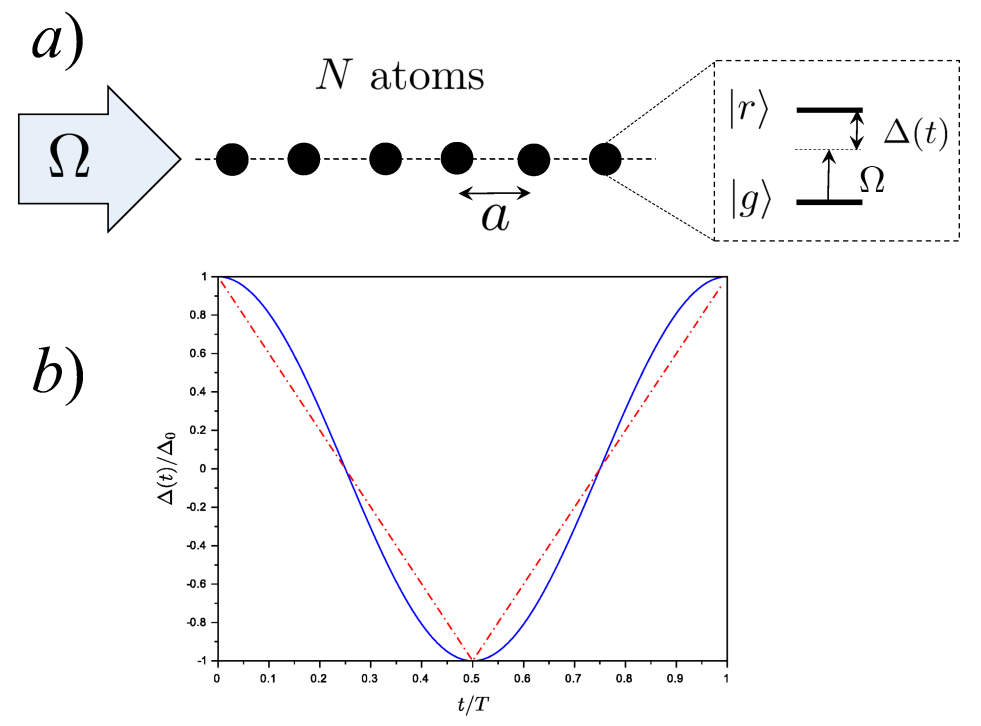

Figure 1. (a) The model system : $N$ atoms with two levels $\{|g\rangle,|r\rangle\}$ are regularly arranged on a line with the step $a$, and submitted to a near-resonant laser field of Rabi frequency $\Omega$ and time-dependent detuning $\Delta(t)$. (b) Typical shapes used for $\Delta(t)$ in simulations (see equations $9,10)$ in the text).

\subsection{Numerical results}

In this section we present the numerical results we obtained for a system of $N=10$ atoms submitted to a frequency-modulated laser beam. We set $\Omega T=2 \pi$ and consider the following two types of modulated detunings (see figure $1 \mathrm{~b}$ )

and

$$
\Delta(t)=\Delta_{0} \times\left\{\begin{array}{c}
1-4\left(\frac{t}{T}-n\right), \text { for } n \leq \frac{t}{T} \leq n+\frac{1}{2} \\
4\left(\frac{t}{T}-n\right)-3, \text { for } n+\frac{1}{2} \leq \frac{t}{T} \leq n+1
\end{array}\right.
$$

$$
\Delta(t)=\Delta_{0} \cos \left(\frac{2 \pi t}{T}\right)
$$

For a given set of parameters $\left\{\Delta_{0} T, C_{6} T / \hbar a^{6}\right\}$ we numerically compute the Floquet evolution $U_{F}$ by splitting the period $[0, T]$ into $K$ short time steps $\tau \equiv \frac{T}{K}$ and resorting to the approximate discretized form

$$
U_{F} \approx e^{-\frac{\mathrm{i} \tau}{\hbar} H(K \tau)} \times \cdots \times e^{-\frac{\mathrm{i} \tau}{\hbar} H(2 \tau)} \times e^{-\frac{\mathrm{i} \tau}{\hbar} H(\tau)}
$$

For the parameters we use in our simulations, we numerically check that $K=100$ steps are enough to obtain a converged value of $U_{F}$; in other words, increasing $K$ beyond 100 does not significantly change $U_{F}$ nor the results obtained, e.g., for the dynamics of populations. The eigenenergies $\hbar \omega_{\alpha}$, eigenstates $|\alpha\rangle$ and the Floquet Hamiltonian $H_{F}$ are derived as described in section 2. Assuming the system is initially in the factorized ground state $\left|\Psi_{0}\right\rangle \equiv|g \cdots g\rangle$, we can straightforwardly compute the stroboscopic evolution of the expectation value of the observable of interest, i.e. the population of the Rydberg level in the ensemble $P_{r}=\sum_{n=1}^{N} \sigma_{r r}^{(n)}$. We set $\mathscr{P}_{r, n} \equiv\left\langle\Psi_{0}\left|\left(U_{F}^{\dagger}\right)^{n} P_{r}\left(U_{F}\right)^{n}\right| \Psi_{0}\right\rangle$. 


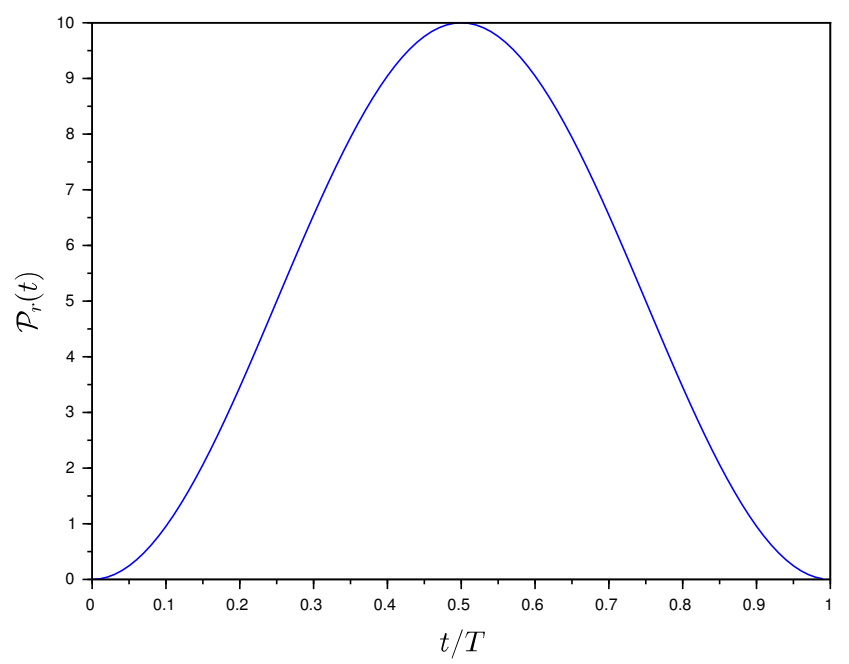

Figure 2. Trivial non-interacting and non-modulated case - The Rydberg population $\mathscr{P}_{r}$ is represented as a function of time on the first period of the drive. The parameters used in this simulation are $\Omega T=2 \pi$ and $C_{6} T / \hbar a^{6}=\Delta_{0} T=0$.

We can also derive the respective relevances $q_{\alpha \beta}$ of the gaps $G_{\alpha \beta}$ for the observable of interest, as well as the thermalised value $\overline{\mathscr{P}}_{r}$ and associated standard deviation $\bar{\sigma}_{r}$.

Before presenting and analysing our results, let us note that our choice for $\left|\Psi_{0}\right\rangle \equiv|g \cdots g\rangle$ corresponds to the typical initial state in Rydberg experiments. This configuration was considered in the first articles on thermalisation in Rydberg atomic systems (see, e.g., [27, 28]). Other choices for $\left|\Psi_{0}\right\rangle=\sum_{\alpha} c_{\alpha}|\alpha\rangle$ should a priori lead to different Floquet thermalised states $\bar{\rho}=\sum_{\alpha}\left|c_{\alpha}\right|^{2}|\alpha\rangle\langle\alpha|$. It was, however, shown that a nonintegrable periodically driven model generically leads to a Floquet thermalised state independent of the initial condition [47]. By contrast to this generic scenario, recent works pointed out the existence of quantum scars in certain Rydberg atomic systems, i.e. eigenstates leading to persistent coherent oscillatory dynamics [48]. According to the preliminary numerical checks we performed on systems with moderate numbers of atoms, starting from randomly picked initial states $\left|\Psi_{0}\right\rangle$ (with one or several Rydberg excitations), our system seems to follow the generic case. But, in the absence of a systematic joint numerical and theoretical study, we cannot claim quantum scars do not exist in our system. This will be the subject of future work.

We start with the trivial case $\frac{C_{6} T}{\hbar a^{6}}=\Delta_{0} T=0$ which corresponds to non-interacting atoms undergoing independent and identical Rabi oscillations at the frequency $2 \pi / \Omega=T$ induced by the resonant laser beam. Figure 2 displays the continuous-time evolution of the population $\mathscr{P}_{r}(t)$ over the first period of the time-dependent detuning (though in this case, the amplitude of modulation is zero): one full oscillation can be clearly identified. Measuring the population $\mathscr{P}_{r}(t)$ at the beginning of each period therefore yields zero and accordingly $\mathscr{P}_{r, n}$ remains constantly zero.

By contrast, figure 3 shows $\mathscr{P}_{r, n}$ over 800 periods for $\Delta_{0} T=5$ and $C_{6} T / \hbar a^{6}=8$ in the case of a triangular $T$-periodic time-dependent detuning $\Delta(t)$ defined in equation (9). Now, one observes a quick equilibration of $\mathscr{P}_{r, n}$ around the average value $\overline{\mathscr{P}}_{r} \approx 4.62$ with reduced long-term fluctuations 


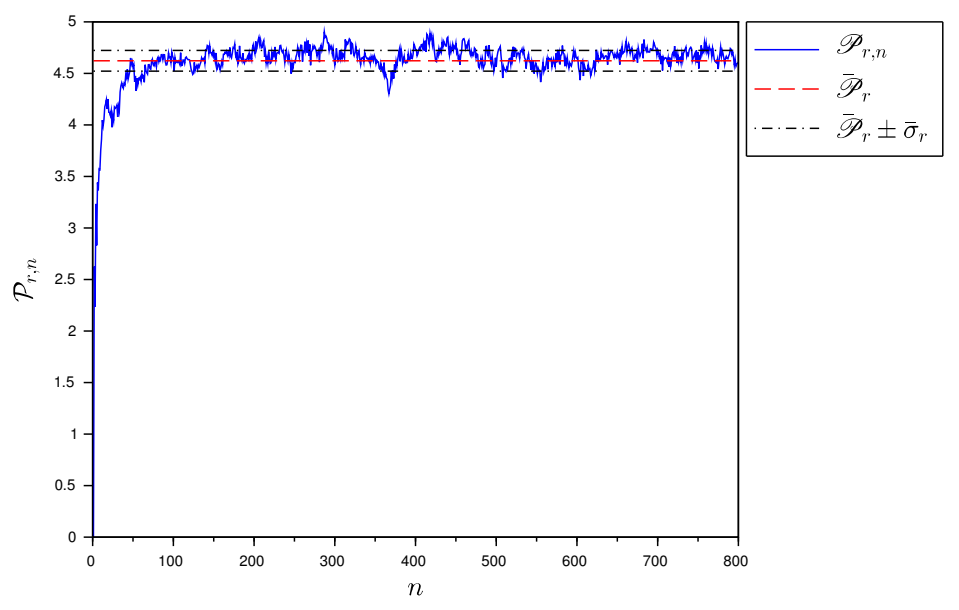

Figure 3. Triangular frequency modulation : Floquet thermalisation of the Rydberg state population - The Rydberg population $\mathscr{P}_{r, n}$ is represented by a full (blue) line, the thermalised value $\overline{\mathscr{P}}_{r}$ by a dashed (red) line, the fluctuation area is delimited by dashed-dotted (black) lines. The parameters used in this simulation are $\Omega T=2 \pi, C_{6} T / \hbar a^{6}=8$ and $\Delta_{0} T=5$.

$\bar{\sigma}_{r} \approx 0.10$. With this choice of parameters, the simultaneous excitation of two neighbouring atoms is highly prevented, i.e. one imposes nearest-neighbour Rydberg blockade, as confirmed by the average number of excited along the chain, $\overline{\mathscr{P}}_{r} \approx N / 2$.

The dynamical behaviour of $\mathscr{P}_{r, n}$ may be qualitatively accounted for through analyzing the distributions of energy gaps and associated relevances of the system, represented in figures 4,5 and 6. We first make two remarks: A. For sake of readibility of figures 4,5 and 6 , we chose not to display : i) degenerate state pairs $(|\alpha\rangle,|\alpha\rangle)$ of zero energy gaps which play no role in the time-dependent part of equation (1) and ii) the state pairs $(|\alpha\rangle,|\beta\rangle)$ of negligible relevances $q_{r, \alpha \beta}$, i.e. for which $q_{r, \alpha \beta} \leq 10^{-6}$. This is why, e.g., only $\approx 3500$ state pairs appear in figure 4 , while the actual number of state pairs in the system is $2^{20} \approx 10^{6}$. B. The relevance distribution is symmetric with respect to the zero energy gap. This results from the reality of the expectation value in equation (1).

We now turn to the interpretation of figures 4,5 and 6 . The relevance distribution on figure 4 can be roughly split into a (dominating) low-frequency and a high-frequency parts, respectively displayed on figures 5 (a) and 6 (a). The boundary between low- and high-frequency contributions was fixed at $\left|G_{\alpha \beta}\right| \approx 0.1 \times \Omega$ which, in order of magnitude, corresponds to $0.1 \times T^{-1}$ for the parameters used in our simulations. Following the (oversimplistic but intuitive) picture of populations performing slowly damped Rabi oscillations, the separation between low and high frequencies thus defined therefore allows us to isolate the slow damping from rapid Rabi oscillations. The associated reduced low-frequency and high-frequency dynamics are represented on figures 5 (b) and 6 (b), respectively, and compared to the exact full dynamics on figures 5 (c) and 6 (c), respectively. Note that the zero-frequency component (associated to degenerate state pairs $(|\alpha\rangle,|\alpha\rangle))$ was implicitly included in the calculation of both low-frequency and high-frequency reduced dynamics to make the comparison with the full dynamics easier.

The low-frequency part is dominated by two symmetric peaks denoted by A on figure 5 (a), of 


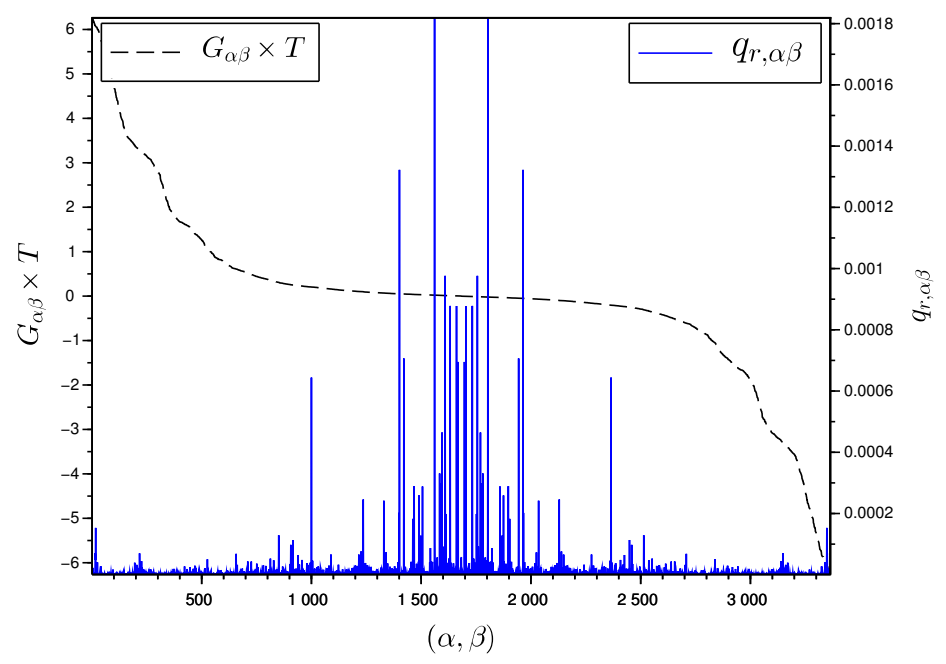

Figure 4. Triangular frequency modulation - Energy gaps $G_{\alpha \beta}$ and associated relevances $q_{r, \alpha \beta}$ are represented by a dashed (black) and a full (blue) lines, respectively. For sake of clarity, the $x$-axis does not show degenerate state pairs $(|\alpha\rangle,|\alpha\rangle)$ of zero energy gaps, nor state pairs $(|\alpha\rangle,|\beta\rangle)$ for which $q_{r, \alpha \beta} \leq 10^{-6}$. The remaining pairs are arbitrarily numbered in such a way that $G_{\alpha \beta}$ 's appear in decreasing order. The parameters used in this simulation are $\Omega T=2 \pi$, $C_{6} T / \hbar a^{6}=8$ and $\Delta_{0} T=5$.

gaps $\pm G_{A} \approx \pm 0.02 \times T^{-1}$, surrounded by a band of gaps of width $\Delta G \approx 0.1 \times T^{-1}$. The associated dynamics consists of very slow damped oscillations of period $2 \pi / G_{A} \approx 292 \times T$ and typical damping timescale $\tau_{\mathrm{d}}=2 \pi / \Delta G \approx 65 \times T$. The two pairs of secondary peaks outside the main band, denoted by $\mathrm{B}$ and $\mathrm{C}$ on 5 (a), induce surviving oscillations of typical period is given by $4 \pi /\left(G_{B}-G_{C}\right) \approx 75 \times T$. This simple analysis is in good qualitative agreement with the dynamics observed on figures 5 (b) and (c).

The high-frequency part is dominated by a pair of symmetric peaks, denoted by A on figure 6 (a), of gaps $\pm G_{A} \approx \pm 0.20 \times T^{-1}$ associated to relatively slow oscillations of period $2 \pi / G_{A} \approx 31 \times T$ which can be qualitatively identified on figures 6 (b) and (c). Because of its complexity, the rest of the distribution does not lend itself to direct interpretation : it comprises many high-frequency peaks which induce fast periodic dynamics of respective periods $\approx 1,2,6,10,20, \cdots \times T$ (among others) and result in the noisy fluctuations observed on figures 6 (b) and (c).

Floquet thermalisation is also accompanied by a synchronization phenomenon: in other words, the continuous-time function $\mathscr{P}_{r}(t)$ tends to synchronize with the drive and becomes itself $T$ periodic. This can be seen on figure 7 which shows superimposed plots of the function $\mathscr{P}_{r}(t)$ on the first (top) and last (bottom) ten periods of the range considered in figure 3: while the behaviour of $\mathscr{P}_{r}(t)$ is quite different on the first ten periods, the traces of $\mathscr{P}_{r}(t)$ on the last ten periods almost coincide.

Figures 8 and 9 analyze the dependence on the parameters $\Delta_{0} T$ and $C_{6} T / \hbar a^{6}$, respectively, of the quantities $\overline{\mathscr{P}}_{r}, \bar{\sigma}_{r}$ and $\bar{\sigma}_{r} / \overline{\mathscr{P}}_{r}$. The latter is particularly significant since it quantifies how effective Floquet thermalisation is. As shown on figures 8 and 9, thermalisation is robust with 
(a)

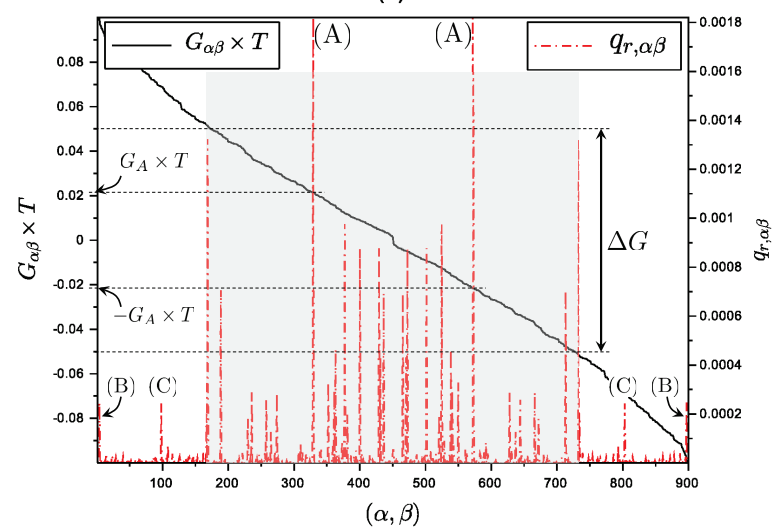

(b)

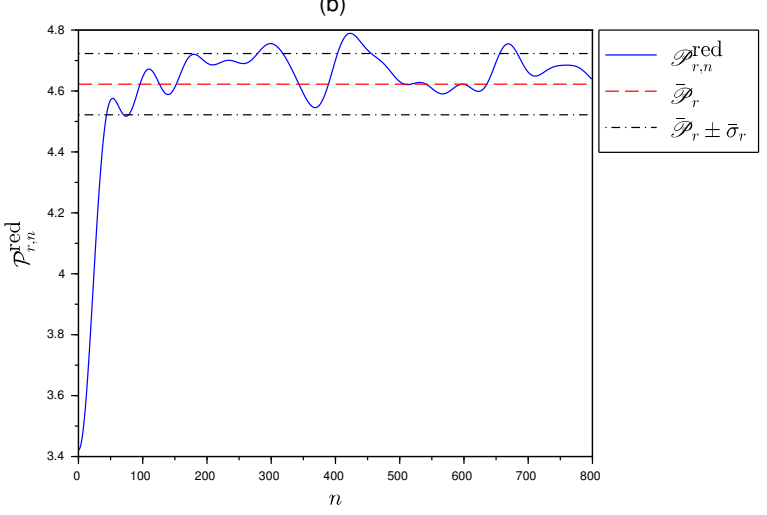

(c)

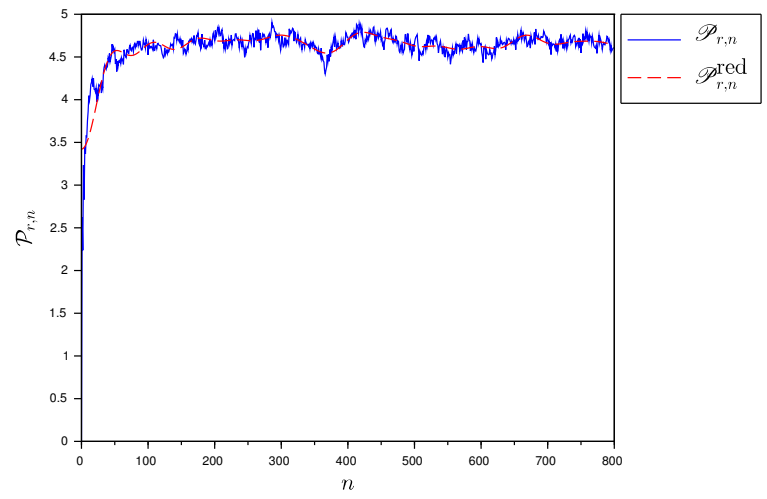

Figure 5. Triangular frequency modulation : low-frequency dynamics - (a) Lowfrequency energy gaps $G_{\alpha \beta}$, i.e. for which $\left|G_{\alpha \beta}\right| \leq 0.1 \times T^{-1}$, and associated relevances $q_{r, \alpha \beta}$ are represented by a full (black) and a dashed (red) lines, respectively. For sake of clarity, the $x$-axis does not show degenerate state pairs $(|\alpha\rangle,|\alpha\rangle)$ of zero energy gaps, nor state pairs $(|\alpha\rangle,|\beta\rangle)$ for which $q_{r, \alpha \beta} \leq 10^{-6}$. The remaining pairs are arbitrarily numbered in such a way that $G_{\alpha \beta}$ 's appear in decreasing order. (b) The reduced stroboscopic dynamics of the Rydberg population $\mathscr{P}_{r, n}^{\text {red }}$ induced by the low-frequency gaps only - including zero-frequency components $G_{\alpha \alpha}$ not displayed on figure (a), is represented by a full (blue) line as a function of the number of periods $n$, the thermalised value $\overline{\mathscr{P}}_{r}$ by a dashed (red) line, the fluctuation area is delimited by dasheddotted (black) lines. (c) The exact and reduced stroboscopic dynamics of the Rydberg population, $\mathscr{P}_{r, n}$ and $\mathscr{P}_{r, n}^{\text {red }}$, are represented by a full (blue) and a dashed (red) lines, respectively, as functions of the number of periods $n$. The parameters used in this simulation are $\Omega T=2 \pi, C_{6} T / \hbar a^{6}=8$ and $\Delta_{0} T=5$. 
(a)

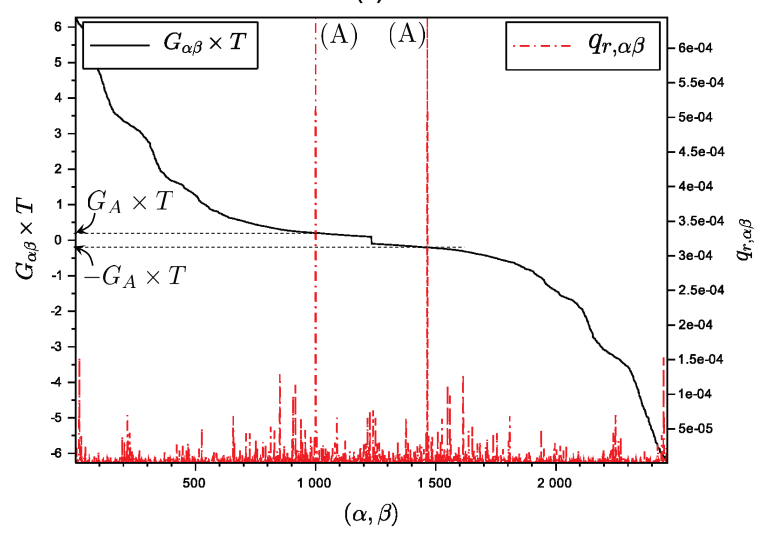

(b)

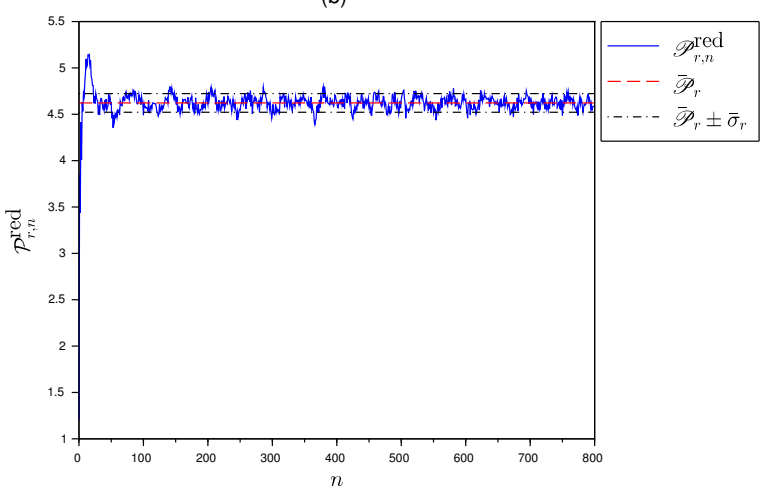

(c)

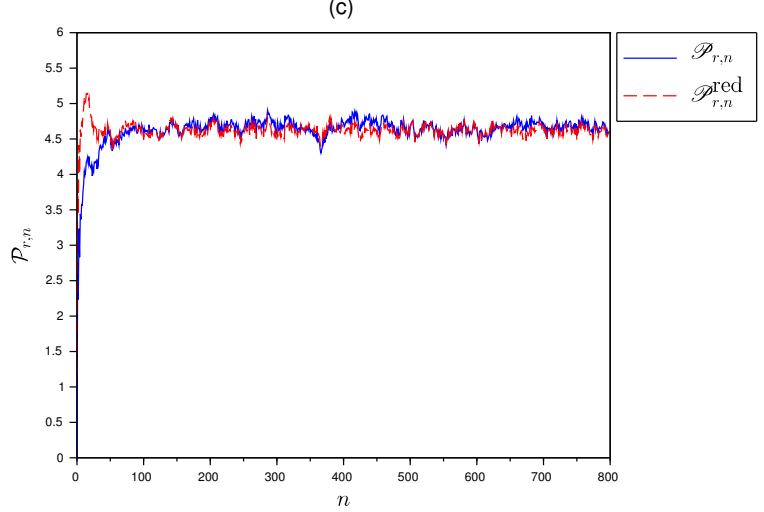

Figure 6. Triangular frequency modulation : high-frequency dynamics - (a) Highfrequency energy gaps $G_{\alpha \beta}$, i.e. for which $\left|G_{\alpha \beta}\right| \geq 0.1 \times T^{-1}$, and associated relevances $q_{r, \alpha \beta}$ are represented by a full (black) and a dashed (red) lines, respectively. For sake of clarity, the $x$-axis does not show degenerate state pairs $(|\alpha\rangle,|\alpha\rangle)$ of zero energy gaps, nor state pairs $(|\alpha\rangle,|\beta\rangle)$ for which $q_{r, \alpha \beta} \leq 10^{-6}$. The remaining pairs are arbitrarily numbered in such a way that $G_{\alpha \beta}$ 's appear in decreasing order. (b) The reduced stroboscopic dynamics of the Rydberg population $\mathscr{P}_{r, n}^{\text {red }}$ induced by the high-frequency gaps only - also including zero-frequency components $G_{\alpha \alpha}$ not displayed on figure (a), is represented by a full (blue) line as a function of the number of periods $n$, the thermalised value $\overline{\mathscr{P}}_{r}$ by a dashed (red) line, the fluctuation area is delimited by dashed-dotted (black) lines. (c) The exact and reduced stroboscopic dynamics of the Rydberg population, $\mathscr{P}_{r, n}$ and $\mathscr{P}_{r, n}^{\text {red }}$, are represented by a full (blue) and a dashed (red) lines, respectively, as functions of the number of periods $n$. The parameters used in this simulation are $\Omega T=2 \pi$, $C_{6} T / \hbar a^{6}=8$ and $\Delta_{0} T=5$. 

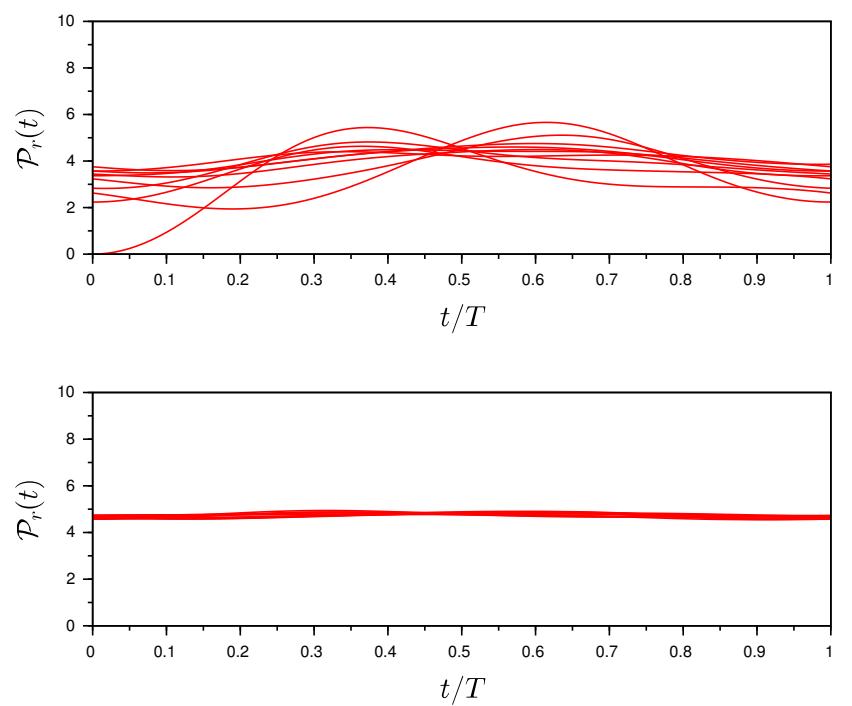

Figure 7. Triangular frequency modulation : Synchronization with the drive - The plots of the continuous-time function $\mathscr{P}_{r}$ over the first (top) and last (bottom) ten periods are superimposed. The parameters used in this simulation are $\Omega T=2 \pi, C_{6} T / \hbar a^{6}=8$ and $\Delta_{0} T=5$.

respect to variations in the system's parameters: in the range of $\Delta_{0} T$ values investigated around $\Delta_{0} T=5, \bar{\sigma}_{r} / \overline{\mathscr{P}}_{r} \leq 0.022$, while, in the range of $C_{6} T / \hbar a^{6}$ values investigated around $C_{6} T / \hbar a^{6}=8$, $\bar{\sigma}_{r} / \overline{\mathscr{P}}_{r} \leq 0.022$.

In the same spirit, one can investigate the robustness of the phenomenon against a slight distortion of the modulation scheme. Our ambition here is not to provide a quantitative and systematic analysis of the influence of the frequency modulation shape on, e.g., the spectral properties of $H_{F}$ and/or the population dynamics, this is much beyond the scope of the present work. More modestly, on the specific example presented below, we merely want to point out that the observed thermalisation phenomenon seems to remain qualitatively unchanged when slightly modifying the modulation scheme.

Figures 10-16 address the case of a $T$-periodic cosine time-dependent detuning, defined in equation (10) with the same parameters $\Omega T=2 \pi, C_{6} T / \hbar a^{6}=8$ and $\Delta_{0} T=5$ as above. The results obtained are very much alike previous ones. Equilibration of $\mathscr{P}_{r, n}$ is observed around its average $\overline{\mathscr{P}}_{r} \approx 4.74$ with reduced long-time fluctuations $\bar{\sigma}_{r} \approx 0.09$ (figure 10 ), as well as synchronization of $\mathscr{P}_{r}(t)$ (figure 14).

As in the triangle-frequency-modulation case, the dynamical behaviour of $\mathscr{P}_{r, n}$ is qualitatively accounted for through analyzing the distributions of energy gaps and associated relevances of the system, represented in figure 11. Again, the relevance distribution on figure 11 can be roughly split into a (dominating) low-frequency and a high-frequency parts, respectively displayed on figures 12 (a) and 13 (a). The associated reduced low-frequency and high-frequency dynamics are represented on figures 12 (b) and 13 (b), respectively, and compared to the exact full dynamics on figures 12 (c) and $13(\mathrm{c})$, respectively.

The low-frequency part is again dominated by two symmetric peaks denoted by A on figure 


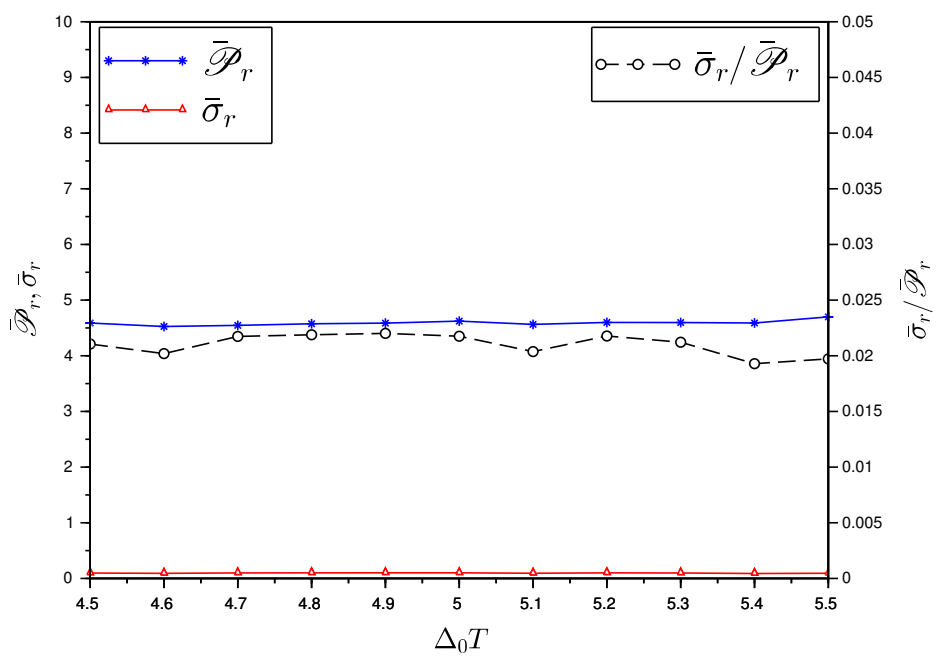

Figure 8. Triangular frequency modulation : influence of the parameter $\Delta_{0} T$ on the thermalisation phenomenon - The thermalised Rydberg population $\overline{\mathscr{P}}_{r}$, standard deviation $\bar{\sigma}_{r}$ and ratio $\bar{\sigma}_{r} / \overline{\mathscr{P}}_{r}$ are represented by (blue) stars, (red) triangles and (black) circles, respectively, as functions of $\Delta_{0} T$. The parameters used in this simulation are $\Omega T=2 \pi$ and $C_{6} T / \hbar a^{6}=8$.

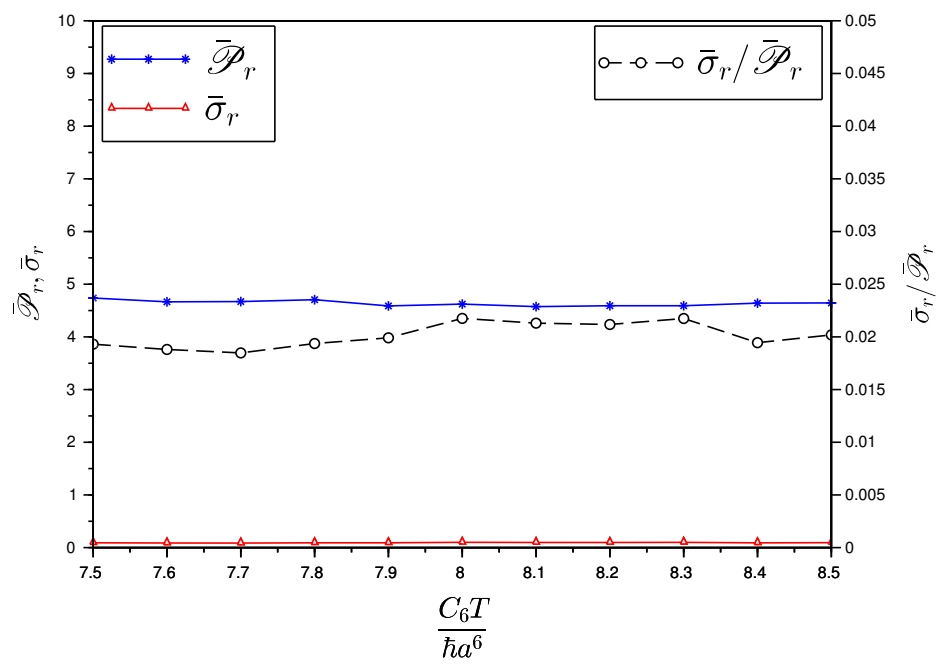

Figure 9. Triangular frequency modulation : influence of the parameter $C_{6} T / \hbar a^{6}$ on the thermalisation phenomenon - The thermalised Rydberg population $\overline{\mathscr{P}}_{r}$, standard deviation $\bar{\sigma}_{r}$ and ratio $\bar{\sigma}_{r} / \overline{\mathscr{P}}_{r}$ are represented by (blue) stars, (red) triangles and (black) circles, respectively, as functions of $C_{6} T / \hbar a^{6}$. The parameters used in this simulation are $\Omega T=2 \pi$ and $\Delta_{0} T=5$. 


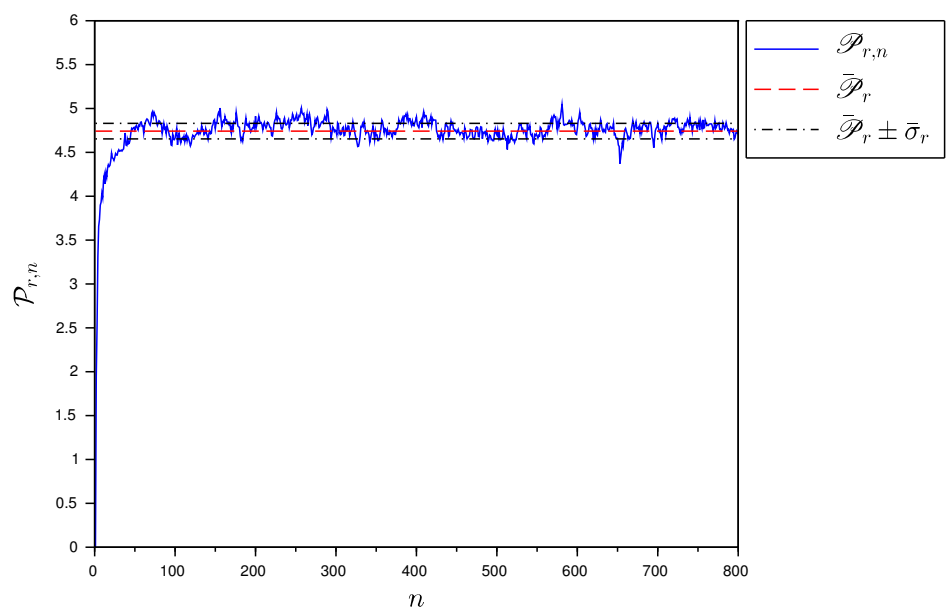

Figure 10. Cosine frequency modulation - Floquet thermalisation of the Rydberg state population. The Rydberg population $\mathscr{P}_{r, n}$ is represented by a full (blue) line, the thermalised value $\overline{\mathscr{P}}_{r}$ by a dashed (red) line, the fluctuation area is delimited by dashed-dotted (black) line. The parameters used in this simulation are $\Omega T=2 \pi, C_{6} T / \hbar a^{6}=8$ and $\Delta_{0} T=5$.

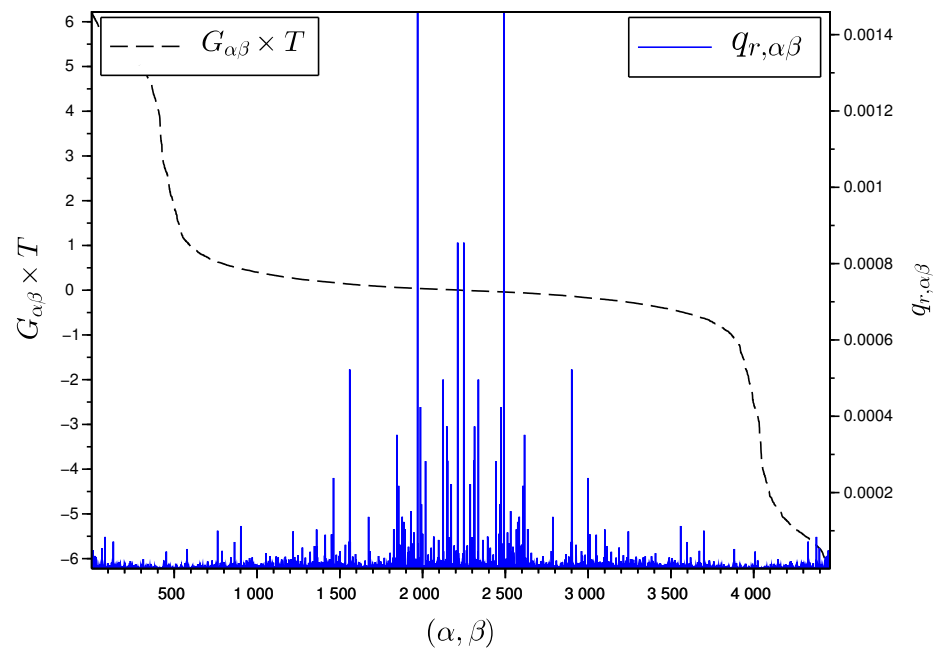

Figure 11. Cosine frequency modulation - Energy gaps $G_{\alpha \beta}$ and associated relevances $q_{r, \alpha \beta}$ are represented by a dashed (black) and a full (blue) lines, respectively. For sake of clarity, the $x$-axis does not show degenerate state pairs $(|\alpha\rangle,|\alpha\rangle)$ of zero energy gaps, nor state pairs $(|\alpha\rangle,|\beta\rangle)$ for which $q_{r, \alpha \beta} \leq 10^{-6}$. The remaining pairs are arbitrarily numbered in such a way that $G_{\alpha \beta}$ 's appear in decreasing order. The parameters used in this simulation are $\Omega T=2 \pi$, $C_{6} T / \hbar a^{6}=8$ and $\Delta_{0} T=5$. 
12 (a), of gaps $\pm G_{A} \approx \pm 0.039 \times T^{-1}$, surrounded by a band of gaps of width $\Delta G \approx 0.094 \times T^{-1}$. The associated dynamics consists of very slow damped oscillations of period $2 \pi / G_{A} \approx 160 \times T$ and typical damping timescale $\tau_{\mathrm{d}}=2 \pi / \Delta G \approx 70 \times T$. The pair of secondary peaks outside the main band, denoted by B on 12 (a) (and peaks close to B), of gaps $\pm G_{B} \approx \pm 0.06 \times T^{-1}$ induce surviving oscillations of typical period by $2 \pi / G_{B} \approx 105 \times T$. Here again our simple analysis reproduces the essential features observed on figures 12 (b) and (c).

The high-frequency part is dominated by a pair of symmetric peaks, denoted by $\mathrm{A}$ on figure 13 (a), of gaps $\pm G_{A} \approx \pm 0.135 \times T^{-1}$ associated to relatively slow oscillations of period $2 \pi / G_{A} \approx 46 \times T$ which can be qualitatively identified on figures 13 (b) and (c). The presence of many secondary peaks makes, however, further analysis illusive: the noisy fluctuations observed on figures 13 (b) and (c) results from the superposition of oscillations with periods $\approx 1,2,6,10,13,17,23, \cdots \times T$ (among others).

Finally figures 15 and 16 show the strong robustness of Floquet thermalisation with respect to changes in the system's parameters $\Delta_{0} T$ and $C_{6} T / \hbar a^{6}$ : in the range of $\Delta_{0} T$ values investigated around $\Delta_{0} T=5, \bar{\sigma}_{r} / \overline{\mathscr{P}}_{r} \leq 0.020$, while, in the range of $C_{6} T / \hbar a^{6}$ values investigated around $C_{6} T / \hbar a^{6}=8, \bar{\sigma}_{r} / \overline{\mathscr{P}}_{r} \leq 0.021$.

\subsection{Experimental implementation}

To close this section we suggest a possible experimental implementation to investigate the Floquet thermalisation identified above. We consider the same configuration as in [45], i.e. rubidium atoms ${ }^{87} \mathrm{Rb}$ with a ground and Rydberg states, respectively $|g\rangle=\left|5 s_{1 / 2} ; F=1 ; m_{F}=1\right\rangle$ and $|r\rangle=$ $\left|60 s ; j=1 / 2 ; m_{j}=1 / 2\right\rangle$, coupled through a two-photon process via the non-resonant intermediate state $|e\rangle=\left|5 p_{1 / 2} ; F=1 ; m_{F}=0\right\rangle$. Adiabatic elimination of $|e\rangle$ [49] yields an effective model of interacting two-level atoms as considered above. The van der Waals coefficient of the Rydberg state is $\frac{C_{6}}{\hbar}=2 \pi \times 1.4 \times 10^{11} \mathrm{~Hz} \cdot \mu \mathrm{m}^{6}$ and its spontaneous decay rate is $\gamma_{\mathrm{r}}=2 \pi \times 2.0 \mathrm{kHz}$.

Setting $T \approx 80 \mathrm{~ns}, \Delta_{0} \approx 2 \pi \times 10 \mathrm{MHz}, \Omega=2 \pi \times 12.5 \mathrm{MHz}$ and $a \approx 4.5 \mu \mathrm{m}$ - which are feasible

parameters in current experiments with atoms trapped in optical tweezers [13] - we get $\frac{C_{6} T}{\hbar a^{6}} \approx 8$ and $\Delta_{0} T=5$ which allow to observe the thermalisation identified above.

Note that with our choice of parameters, $\gamma_{\mathrm{r}} T \approx 10^{-3}$. For approximately the first hundred periods, spontaneous emission will therefore have no noticeable influence on the dynamics of the system. This is sufficient for the system to reach the thermal regime and therefore exhibit Floquet thermalisation and synchronization in both triangle and cosine frequency modulation schemes studied here. The analysis of the impact of spontaneous emission is, however, important since it may compete with the phenomena investigated here and lead to qualitatively different phenomena in the long-time limit. It will be addressed in a future work.

\section{Conclusion}

In this article we investigated the emergence of Floquet thermalisation and synchronization in a chain of atoms excited by a frequency-modulated laser beam on a transition towards a Rydberg state. We related this phenomenon to properties of the Floquet Hamiltonian spectrum, i.e. the distribution of energy gaps contributing to the Rydberg population dynamics. We found this process is robust against small variations of the parameters, such as the detuning modulation amplitude or the distance between atoms. Finally we suggested a possible experimental situation in which Rydberg-blockade induced Floquet thermalisation could be investigated. 
(a)

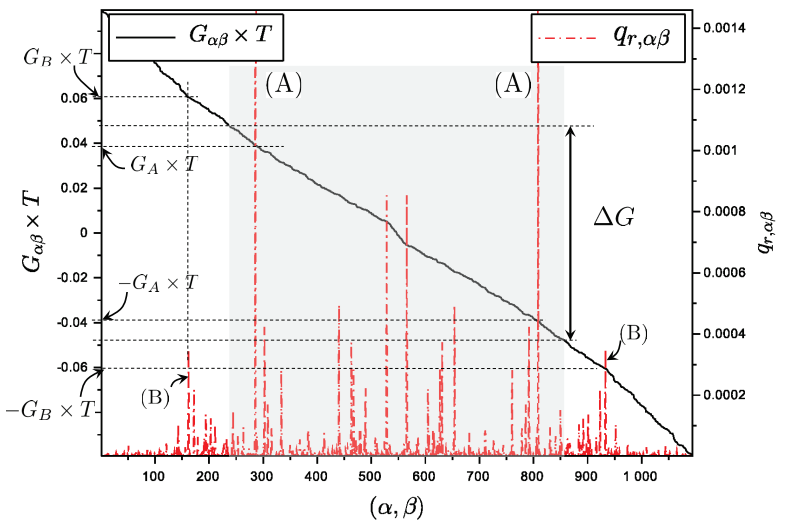

(b)

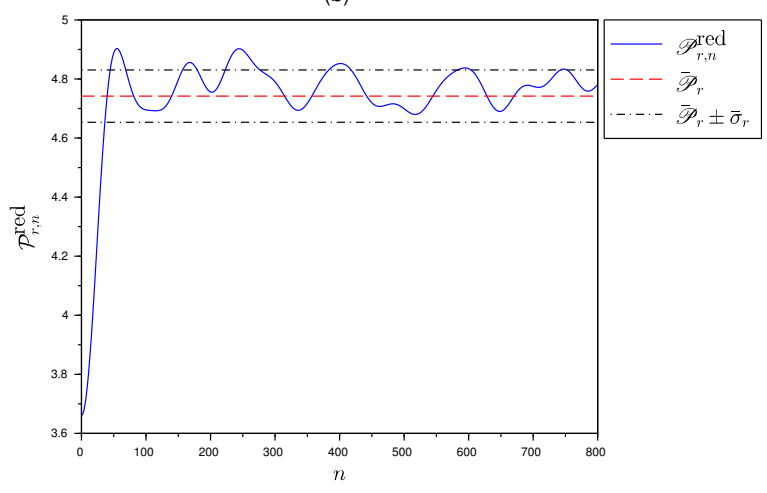

(c)

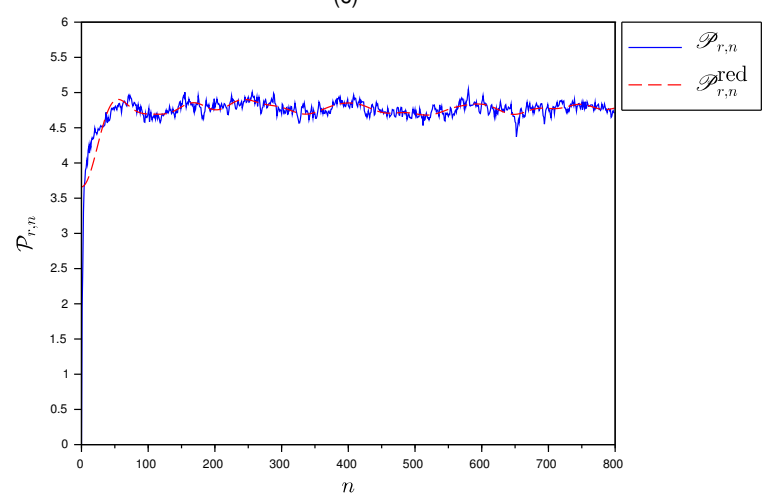

Figure 12. Cosine frequency modulation : low-frequency dynamics - (a) Low-frequency energy gaps $G_{\alpha \beta}$, i.e. for which $\left|G_{\alpha \beta}\right| \leq 0.1 \times T^{-1}$, and associated relevances $q_{r, \alpha \beta}$ are represented by a full (black) and a dashed (red) lines, respectively. For sake of clarity, the $x$-axis does not show degenerate state pairs $(|\alpha\rangle,|\alpha\rangle)$ of zero energy gaps, nor state pairs $(|\alpha\rangle,|\beta\rangle)$ for which $q_{r, \alpha \beta} \leq 10^{-6}$. The remaining pairs are arbitrarily numbered in such a way that $G_{\alpha \beta}$ 's appear in decreasing order. (b) The reduced stroboscopic dynamics of the Rydberg population $\mathscr{P}_{r, n}^{\text {red }}$ induced by the low-frequency gaps only - including zero-frequency components $G_{\alpha \alpha}$ not displayed on figure (a), is represented by a full (blue) line as a function of the number of periods $n$, the thermalised value $\overline{\mathscr{P}}_{r}$ by a dashed (red) line, the fluctuation area is delimited by dashed-dotted (black) lines. (c) The exact and reduced stroboscopic dynamics of the Rydberg population, $\mathscr{P}_{r, n}$ and $\mathscr{P}_{r, n}^{\text {red }}$, are represented by a full (blue) and a dashed (red) lines, respectively, as functions of the number of periods $n$. The parameters used in this simulation are $\Omega T=2 \pi, C_{6} T / \hbar a^{6}=8$ and $\Delta_{0} T=5$. 
(a)

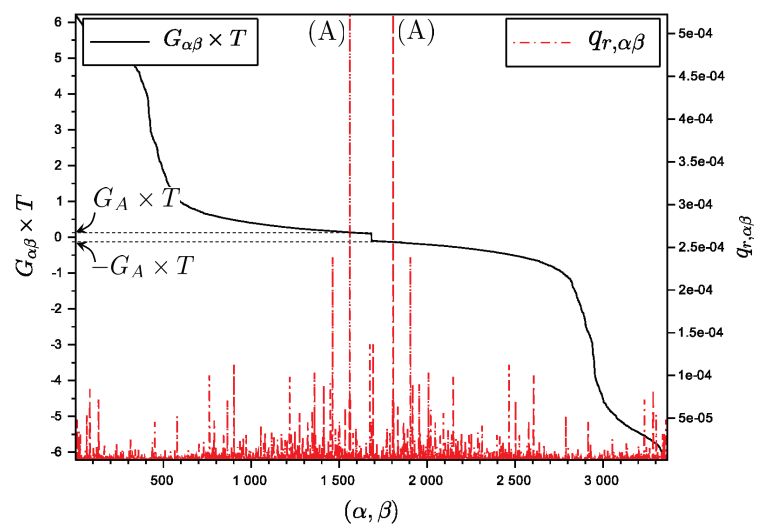

(b)

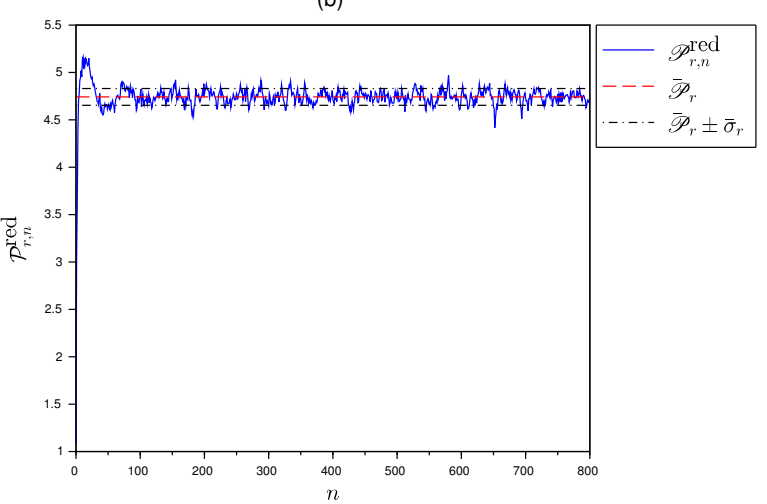

(c)

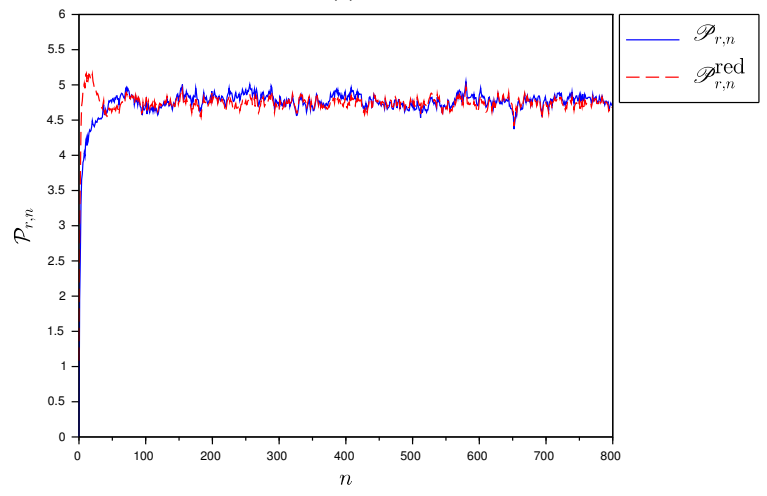

Figure 13. Cosine frequency modulation : high-frequency dynamics - (a) Highfrequency energy gaps $G_{\alpha \beta}$, i.e. for which $\left|G_{\alpha \beta}\right| \geq 0.1 \times T^{-1}$, and associated relevances $q_{r, \alpha \beta}$ are represented by a full (black) and a dashed (red) lines, respectively. For sake of clarity, the $x$-axis does not show degenerate state pairs $(|\alpha\rangle,|\alpha\rangle)$ of zero energy gaps, nor state pairs $(|\alpha\rangle,|\beta\rangle)$ for which $q_{r, \alpha \beta} \leq 10^{-6}$. The remaining pairs are arbitrarily numbered in such a way that $G_{\alpha \beta}$ 's appear in decreasing order. (b) The reduced stroboscopic dynamics of the Rydberg population $\mathscr{P}_{r, n}^{\text {red }}$ induced by the high-frequency gaps only - also including zero-frequency components $G_{\alpha \alpha}$ not displayed on figure (a), is represented by a full (blue) line as a function of the number of periods $n$, the thermalised value $\overline{\mathscr{P}}_{r}$ by a dashed (red) line, the fluctuation area is delimited by dashed-dotted (black) lines. (c) The exact and reduced stroboscopic dynamics of the Rydberg population, $\mathscr{P}_{r, n}$ and $\mathscr{P}_{r, n}^{\text {red }}$, are represented by a full (blue) and a dashed (red) lines, respectively, as functions of the number of periods $n$. The parameters used in this simulation are $\Omega T=2 \pi$, $C_{6} T / \hbar a^{6}=8$ and $\Delta_{0} T=5$. 

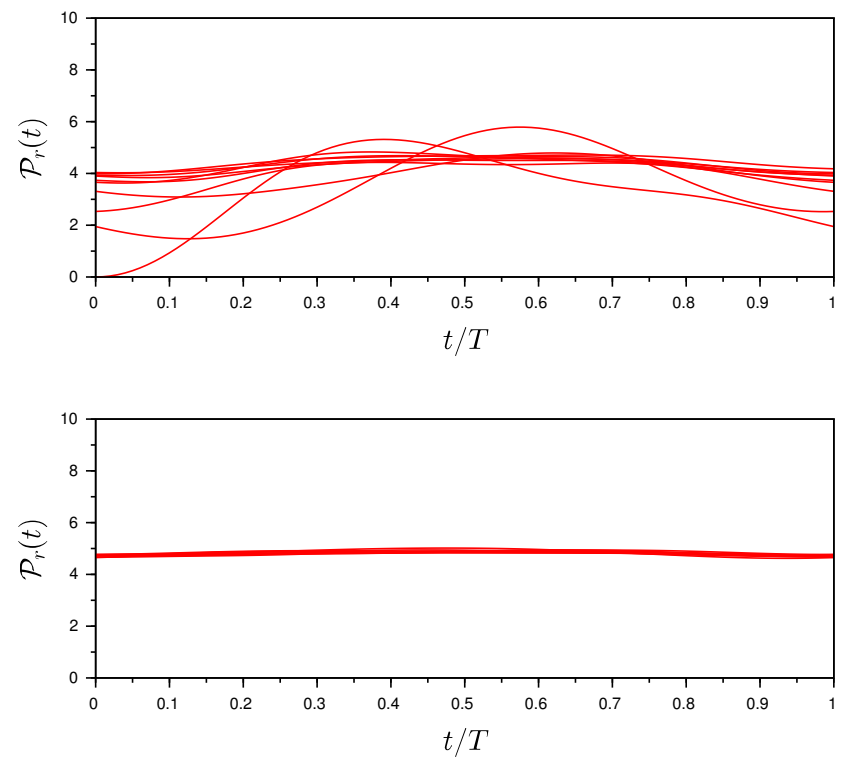

Figure 14. Cosine frequency modulation: Synchronization with the drive - The plots of the continuous-time function $\mathscr{P}_{r}$ over the first (top) and last (bottom) ten periods are superimposed. The parameters used in this simulation are $\Omega T=2 \pi, C_{6} T / \hbar a^{6}=8$ and $\Delta_{0} T=5$.

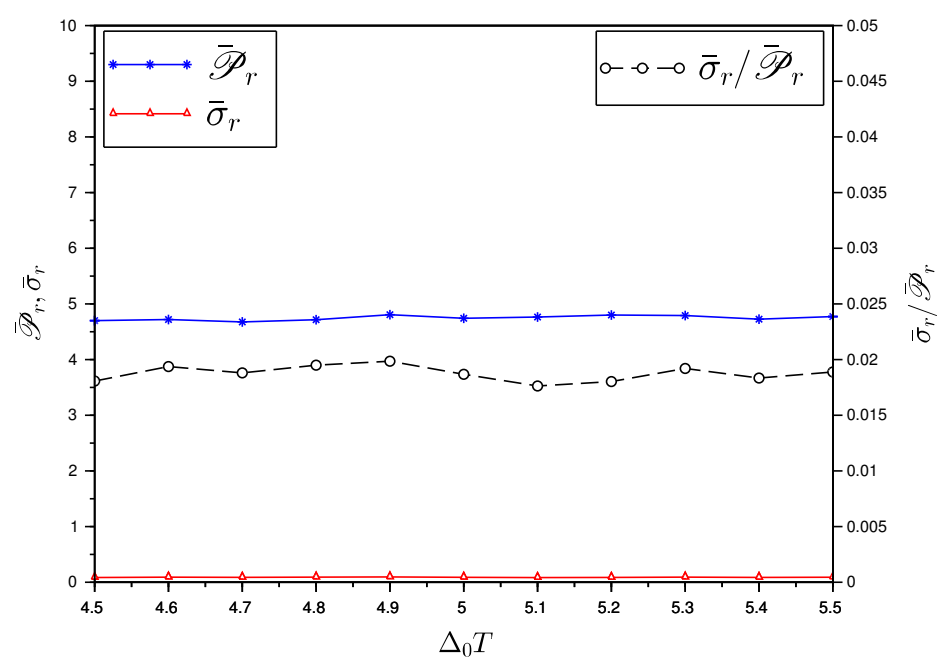

Figure 15. Cosine frequency modulation : influence of the parameter $\Delta_{0} T$ on the thermalisation phenomenon - The thermalised Rydberg population $\overline{\mathscr{P}}_{r}$, standard deviation $\bar{\sigma}_{r}$ and ratio $\bar{\sigma}_{r} / \overline{\mathscr{P}}_{r}$ are represented by (blue) stars, (red) triangles and (black) circles, respectively, as functions of $\Delta_{0} T$. The parameters used in this simulation are $\Omega T=2 \pi$ and $C_{6} T / \hbar a^{6}=8$. 


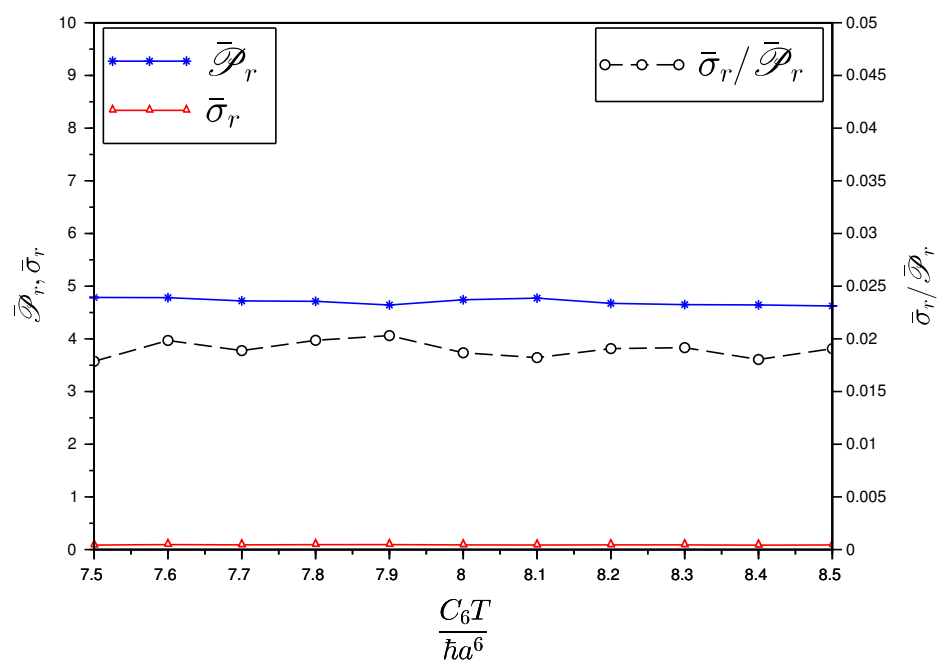

Figure 16. Cosine frequency modulation : influence of the parameter $C_{6} T / \hbar a^{6}$ on the thermalisation phenomenon - The thermalised Rydberg population $\overline{\mathscr{P}}_{r}$, standard deviation $\bar{\sigma}_{r}$ and ratio $\bar{\sigma}_{r} / \overline{\mathscr{P}}_{r}$ are represented by (blue) stars, (red) triangles and (black) circles, respectively, as functions of $C_{6} T / \hbar a^{6}$. The parameters used in this simulation are $\Omega T=2 \pi$ and $\Delta_{0} T=5$.

The impact of unavoidable spontaneous emission from the Rydberg level remains to be systematically investigated. The influence of the spatial arrangement of the atoms on the phenomenon is also worth studying, in particular the choice of other geometries than the line or the introduction of disorder in the atomic positions. Richer level configurations involving several Rydberg states could also be considered, such as, e.g., the excitation of a Förster resonance. These aspects shall be considered in future works.

\section{Acknowledgments}

E. B. thanks F. Alet for helpful discussions.

* retired, previously at the Groupe de Spectrométrie Moléculaire et Atmosphérique, UMR CNRS 7331, University of Reims Champagne-Ardenne, F-51687, Reims Cedex 2, France.

\section{References}

[1] T. F. Gallagher, Rydberg atoms, Cambridge University Press (2005).

[2] M. Saffman, T. G. Walker, and K. Mølmer, Rev. Mod. Phys. 82, 2313 (2010).

[3] D. Comparat and P. Pillet, J. Opt. Soc. Am. B 27, A208 (2010).

[4] D. Jaksch, J. I. Cirac, P. Zoller, S. L. Rolston, R. Côté, and M. D. Lukin, Phys. Rev. Lett. 85, 2208 (2000);

[5] M. D. Lukin, M. Fleischhauer, R. Côté, L. M. Duan, D. Jaksch, J. I. Cirac, and P. Zoller, Phys. Rev. Lett. 87, 037901 (2001). 
[6] I. E. Protsenko, G. Reymond, N. Schlosser, and P. Grangier, Phys. Rev. A 66, 062306 (2002).

[7] A. Gaëtan, Y. Miroshnychenko, T. Wilk, A. Chotia, M. Viteau, D. Comparat, P. Pillet, A. Browaeys, and P. Grangier, Nature Physics 5, 115 (2009).

[8] E. Brion, K. Mølmer, and M. Saffman Phys. Rev. Lett. 99, 260501 (2007).

[9] E. Brion, L. H. Pedersen, M. Saffman, and K. Mølmer Phys. Rev. Lett. 100, 110506 (2008).

[10] B. Zhao, M. Müller, K. Hammerer, and P. Zoller, Phys. Rev. A 81, 052329 (2010).

[11] Y. Han, B. He, K. Heshami, C.-Z. Li, and C. Simon, Phys. Rev. A 81, 052311 (2010).

[12] E. Brion, F. Carlier, V. M. Akulin, and K. Mølmer Phys. Rev. A 85, 042324 (2012).

[13] H. Labuhn, D. Barredo, S. Ravets, S. de Léséleuc, T. Macrì, T. Lahaye \& A. Browaeys, Nature 534, 667 (2016).

[14] H. Bernien, S. Schwartz, A. Keesling, H. Levine, A. Omran, H. Pichler, S. Choi, A. S. Zibrov, M. Endres, M. Greiner, V. Vuletić, M. D. Lukin, Nature 551, 579 (2017).

[15] A. V. Gorshkov, J. Otterbach, M. Fleischhauer, T. Pohl, and M.D. Lukin, Phys. Rev. Lett. 107, $133602(2011)$.

[16] T. Peyronel, O. Firstenberg, Q.-Y. Liang, S. Hofferberth, A. V. Gorshkov, T. Pohl, M.D. Lukin, and V. Vuletić, Nature (London) 488, 57 (2012).

[17] V. Parigi, E. Bimbard, J. Stanojevic, A.J. Hilliard, F. Nogrette, R. Tualle-Brouri, A. Ourjoumtsev, and P. Grangier, Phys. Rev. Lett. 109, 233602 (2012).

[18] O. Firstenberg, T. Peyronel, Q.-Y. Liang, A. V. Gorshkov, M. D. Lukin, and V. Vuletić, Nature (London) 502, 71 (2013).

[19] D. Maxwell, D. J. Szwer, D. Paredes-Barato, H. Busche, J. D. Pritchard, A. Gauguet, K. J. Weatherill, M.P. A. Jones, and C. S. Adams, Phys. Rev. Lett. 110, 103001 (2013).

[20] A. V. Gorshkov, R. Nath, and T. Pohl, Phys. Rev. Lett. 110, 153601 (2013).

[21] A. Grankin, E. Brion, E. Bimbard, R. Boddeda, I. Usmani, A. Ourjoumtsev, and P. Grangier, New J. Phys. 16, 043020 (2014).

[22] H. Gorniaczyk, C. Tresp, J. Schmidt, H. Fedder, and S. Hofferberth, Phys. Rev. Lett. 113, 053601 (2014).

[23] D. Tiarks, S. Baur, K. Schneider, S. Dürr, and G. Rempe, Phys. Rev. Lett. 113, 053602 (2014).

[24] A. Grankin, E. Brion, E. Bimbard, R. Boddeda, I. Usmani, A. Ourjoumtsev and P. Grangier, Phys. Rev. A 92, 043841 (2015).

[25] R. Boddeda, I. Usmani, E. Bimbard, A. Grankin, A. Ourjoumtsev, E. Brion, and P. Grangier, J. Phys. B 49, 084005 (2016).

[26] A. Grankin, E. Brion, R. Boddeda, S. Ćuk, I. Usmani, A. Ourjoumtsev and P. Grangier, Phys. Rev. Lett. 117, 253602 (2016).

[27] B. Olmos, M. Müller and I. Lesanovsky, New Journal of Physics 12, 013024 (2010).

[28] I. Lesanovsky, B. Olmos, and J. P. Garrahan, Phys. Rev. Lett. 105, 100603 (2010).

[29] M. Srednicki, Phys. Rev. E 50, 888 (1994).

[30] J. M. Deutsch, Phys. Rev. A 43, 2046 (1991).

[31] M. A. Cazalilla, A. Iucci, and M.-C. Chung, Phys. Rev. E 85, 011133 (2012).

[32] M. Rigol, V. Dunjko, V. Yurovsky, and M. Olshanii, Phys. Rev. Lett. 98, 050405 (2007). 
[33] P. Calabrese, F. H. L. Essler, and M. Fagotti, Phys. Rev. Lett. 106, 227203 (2011).

[34] M. Fagotti and F. H. L. Essler, Phys. Rev. B 87, 245107 (2013).

[35] Marcos Rigol, Vanja Dunjko and Maxim Olshanii, Nature volume 452, 854 (2008).

[36] T. R. de Oliveira, C. Charalambous, D. Jonathan, M. Lewenstein and A. Riera, New Journal of Physics 20, 033032 (2018).

[37] A. Lazarides, A. Das, and R. Moessner, Phys. Rev. Lett. 112, 150401 (2014).

[38] K. Seetharam, P. Titum, M. Kolodrubetz, and G. Refael, Phys. Rev. B 97, 014311 (2018).

[39] A. Herrmann, Y. Murakami, M. Eckstein, P. Werner, Europhys. Lett. 120, 57001 (2018).

[40] S. A. Weidinger and M. Knap, Sci. Rep. 7, 45382 (2017).

[41] D. V. Else, B. Bauer, and C. Nayak Phys. Rev. Lett. 117, 090402 (2016).

[42] T. Oka, and H. Aoki, 2009, Phys. Rev. B 79, 081406 (2009).

[43] G. Jotzu, M. Messer, R. Desbuquois, M. Lebrat, T. Uehlinger, D. Greif, and T. Esslinger, Nature (London) 515, 237 (2014).

[44] T. Ozawa, H. M. Price, A. Amo, N. Goldman, M. Hafezi, L. Lu, M. C. Rechtsman, D. Schuster, J. Simon, O. Zilberberg, and I. Carusotto, Rev. Mod. Phys. 91, 015006 (2019).

[45] C. Fan, D. Rossini, H.-X. Zhang, J.-H. Wu, M. Artoni, G. C. La Rocca, arXiv:1907.03446 [quant-ph] (2019).

[46] L. W. Clark, N. Jia, N. Schine, C. Baum, A. Georgakopoulos \& J. Simon, Nature 571, 532 (2019).

[47] A. Lazarides, A. Das, and R. Moessner, Phys. Rev. E 90, 012110 (2014).

[48] B. Mukherjee, S. Nandy, A. Sen, D. Sen, and K. Sengupta, arxiv:1907.08212.

[49] E. Brion, L. H. Pedersen and K. Mølmer, J. Phys. A: Math. Theor. 40, 1033 (2007). 